\title{
A Gene Cluster for the Biosynthesis of Dibenzodioxocinons in the Endophyte Pestalotiopsis microspora, a Taxol Producer's
}

\author{
Yanjie Liu ${ }^{1}$, Longfei Chen ${ }^{2,3}$, Qiaohong $\mathrm{Xie}^{1}{ }^{1}, \mathrm{Xi} \mathrm{Yu}^{3}$, Anqing Duan ${ }^{1}$, Yamin Lin ${ }^{1}$, Biyun Xiang ${ }^{1}$, Xiaoran Hao ${ }^{1}$, \\ Wanwan Chen ${ }^{1,4}$, and Xudong $\mathrm{Zhu}^{1 *}$ \\ ${ }^{1}$ Beijing Key Laboratory of Genetic Engineering Drug and Biotechnology, Institute of Biochemistry and Biotechnology, College of Life \\ Sciences, Beijing Normal University, Beijing, 100875, P.R. China \\ ${ }^{2}$ Zhejiang Medicine Co., Ltd., Zhejiang, P.R. China \\ ${ }^{3}$ Department of Microbiology, College of Life Sciences, Nankai University, Tianjin, 300071, P.R. China \\ ${ }^{4}$ Shanghai Fudan-Zhangiiang Bio-Pharmaceutical Co., Ltd., Shanghai, P.R. China
}

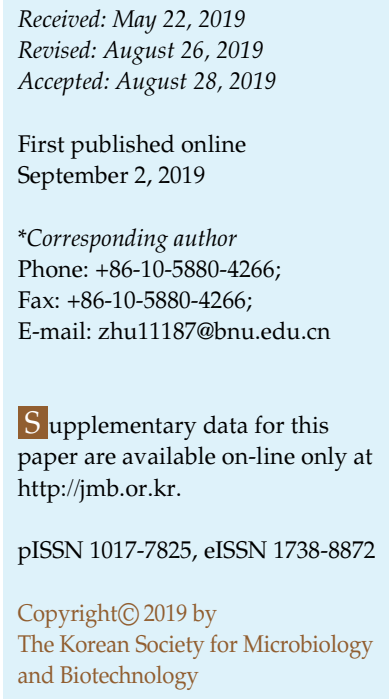

The fungal products dibenzodioxocinones promise a novel class of inhibitors against cholesterol ester transfer protein (CEPT). Knowledge as to their biosynthesis is scarce. In this report, we characterized four more dibenzodioxocinones, which along with a previously described member pestalotiollide $B$, delimit the dominant spectrum of secondary metabolites in $P$. microspora. Through mRNA-seq profiling in $g \alpha 1 \Delta$, a process that halts the production of the dibenzodioxocinones, a gene cluster harboring 21 genes including a polyketide synthase, designated as pks8, was defined. Disruption of genes in the cluster led to loss of the compounds, concluding the anticipated role in the biosynthesis of the chemicals. The biosynthetic route to dibenzodioxocinones was temporarily speculated. This study reveals the genetic basis underlying the biosynthesis of dibenzodioxocinone in fungi, and may facilitate the practice for yield improvement in the drug development arena.

Keywords: Dibenzodioxocinones, CEPT inhibitor, Pestalotiollide B, polyketide synthase, Pestalotiosis microspora NK17

\section{Introduction}

Endophytic fungi isolated from plant hosts have drawn attention from scientists for their ample capacity in making bioactive compounds which are often favorable lead chemicals for drug development [1, 2]. The number of such sourced compounds possessing the bioactivities of antibiotics, immunosuppressants, toxins, and antiviral or anti-tumor agents, keeps climbing [3-6]. Moreover, some so-called secondary metabolites such as these play a critical part during fungal morphogenesis and confer advantages on the fungal host over competitors in nature's niche [7], though, for most of them, their cellular and ecological functions remain largely unclear. Dibenzodioxocinones, virtually a group of polyketides synthesized by fungi, are hallmark in the structure of an alkyl side chain (Figs. 1A and $1 \mathrm{~B}$ ) attached to the core of two benzenes plus a lactonic ring (Fig. 1). Owing to their inhibitory activity against cholesterol ester transfer protein (CETP), they have promising application in the treatment of coronary heart disease (CHD) by raising the levels of high-density lipoprotein cholesterol (HDL-C) [8]. To date, many dibenzodioxocinone derivatives have been isolated and characterized in endophyte fungi such as in the genera Pestalotiopsis [9-11] and Penicillium [12-14]. Unfortunately, the genetic basis governing their biosynthesis has barely been investigated.

Pestalotiopsis microspora NK17 was previously isolated by our laboratory for producing both dibenzodioxocinones and Taxol, the anti-tumor drug $[9,15,16]$. In the genome of NK17, more than thirty gene clusters, putatively participating in secondary metabolism, were demarcated (unpublished data). Pestalotiollide B (1), the first of dibenzodioxocinone analog in P. microspora, was structurally characterized [9] at a remarkable level (Fig. 1B). Thirty-six of a total number of 
A

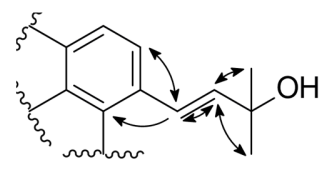

compound $\mathbf{3}$

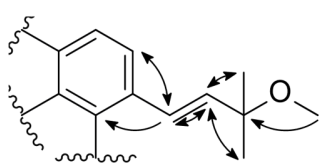

compound 4
B<smiles>COc1c(C)ccc2c1C(=O)OCc1cc(C)cc(O)c1O2</smiles>
1', 2'-dehydropenicillide (3)

3'-methoxy-1', 2'dehydropenicillide (4)

1', 2'-epoxy-3', 4'didehydropenicillide (5)<smiles>C=C(C)[C@H](O)[C@@H](O)[13CH2]C</smiles><smiles>C=C(C)C(O)C(C)O</smiles><smiles>[3H]C([3H])(O)C=CCC</smiles><smiles>CC=CC(C)(C)OC</smiles><smiles>C=C(C)C1OC1CC</smiles><smiles></smiles><smiles>CC1=CC2Cc3cc(O)cc(O)c3C(=O)C2C(O)=C1C(=O)O</smiles><smiles>CC1=CC2C(=O)c3cc(O)cc(O)c3C(=O)C2C(O)=C1C(=O)O</smiles>

endocrocin<smiles>COc1c(C(C)(C)C)ccc(Oc2c(O)cc(C)cc2C=O)c1C(=O)O</smiles><smiles>COc1c(C(C)(C)C)ccc(Oc2c(O)cc(C)cc2CO)c1C(=O)O</smiles>

hydroxytenellic acid B

Fig. 1. Dibenzodioxocinones identified in P. microspora NK17 and some analogs from other fungi.

(A) HMBC signals of the side chain of compound 3 and 4. (B) Compound 1 5 were isolated from NK17. The structure of atrochrysone carboxylic acid was isolated from Pestalotiopsis fici [22], endocrocin anthrone and endocrocin were isolated from Aspergillus nidulans [23], tenellic acid B and hydroxytenellic acid B were from Penicillium sp. MA-37 [14].

41 polyketide synthase (PKS)-encoding genes in the genome of NK17 were individually deleted to distinguish the ones involved in pestalotiollide B production [17]. Nine $p k s$ mutants showed a significant increase in the production of pestalotiollide $B$, while the other six showed an apparent decrease. Noteworthy, the disruption of ga1, encoding group I G protein $\alpha$ subunit, caused a sharp fall in pestalotiollide B production [18], whereas disrupting hid1, a putative histone deacetylase gene, increased pestalotiollide B production by nearly 2-fold [9].

To continue the effort, we herein report the characterization of the major secondary metabolites of $P$. microspora, additional to pestalotiollide B (1), i.e. as shown in Fig. 1, pestalotiollide C (2), 1',2'-dehydropenicillide (3), 3'methoxy-1'2'-dehydropenicillide (4) and $1^{\prime}, 2^{\prime}$-epoxy-3', $4^{\prime}$ didehydropenicillide (5) [12]. Interestingly, all four are dibenzodioxocinone derivatives. Relying on the defective mutant strain $g a 1 \Delta$, a profiling approach by RNA-seq on the genome level was conducted, which helped to successfully identify a gene cluster that we demonstrated to be in charge of the biosynthesis of dibenzodioxocinones. The chemical reactions leading to the formation of dibenzodioxocinones were speculated.

\section{Materials and Methods}

\section{Strains and Plasmids}

The endophyte $P$. microspora strain NK17 was isolated and maintained by our laboratory. All strains, the wild type and the mutants, were routinely grown in potato lactose broth (PLB) or PLA (PLB with $2 \%$ agar) at $28^{\circ} \mathrm{C}$. Plasmid pOSCAR carried in Escherichia coli DB3.1 was used for Agrobacterium tumefaciensmediated transformation (ATMT). Other plasmids were propagated in E. coli $\mathrm{DH} 5 \alpha$. All E. coli strains were cultured in $\mathrm{LB}$ at $37^{\circ} \mathrm{C}$ supplemented with proper antibiotics.

\section{Extraction, Isolation and Characterization of the Compounds}

Fungal strains were grown in $100 \mathrm{ml}$ PLB for 7 days, or on $40 \mathrm{~g}$ sweet potato waste for 13 days at room temperature. The liquid 
culture or the solid substrate with the mycelium was extracted for 3 times by equal volume of ethyl acetate, at room temperature for $24 \mathrm{~h}$ each time. The extracts were condensed in a rotary evaporator at $45^{\circ} \mathrm{C}$ to a volume of approximately $1 \mathrm{ml}$, and were transferred to a $1.5 \mathrm{ml} \mathrm{EP}$ tube for thin-layer chromatography (TLC) display and to determine the polarity for purification step by silica gel column. The mobile phase in TLC was a solvent of dichloromethane and ethyl acetate at a ratio of 7 to 3 . Under this condition, the small chemicals of metabolites were manifestly separated from melanin [19]. Consequently, the crude extract was subjected to silica gel column chromatography for primary purification. We acquired a $4.0 \mathrm{~g}$ mixture of extracts in total. A semi-preparative reversed-phase high performance liquid chromatography (HPLC) (methanol: $\mathrm{H}_{2} \mathrm{O}=7: 3,2 \mathrm{ml} / \mathrm{min}, 227 \mathrm{~nm}$ ) was employed for further purification of the compounds. Four compounds at the retention points of $3.653 \mathrm{~min}$ (1), $3.950 \mathrm{~min}$ (2), $5.363 \mathrm{~min}$ (3), and $9.040 \mathrm{~min}$ (4), respectively, were obtained. And one compound was isolated from the solid culture at the retention point of $10.620 \mathrm{~min}$ (5) (Fig. S1). We obtained 25.9, 34.2, 12.6, 7.6, and $5.5 \mathrm{mg}$ of compound 1-5, respectively.

\section{Total RNA Preparation and RNA-Seq Analysis}

Fungal strains were grown in $100 \mathrm{ml} \mathrm{PLB}$ at $28^{\circ} \mathrm{C}, 200 \mathrm{rpm}$, for 3 days and 6 days, respectively. Total RNA was prepared from lyophilized mycelia using the TRIzol Kit (Invitrogen, Carlsbad, United States). To remove possible contaminant DNA, the RNA samples were treated with RNase-free DNase (Takara, China). RNA-seq and the bioinformatics analysis were carried out by the Beijing Genomics Institute (BGI, China).

\section{Targeted Gene Disruption Via ATMT}

The primers used for targeted disruption of genes in dibenzodioxocinone biosynthesis cluster are listed in Table S1. The methods and steps were carried out according to the protocols described by Chen et al. [17].

\section{Growth of the Targeted Mutants}

The wild type and the mutant strains were cultured on PLA plate or in PLB broth to test whether the targeted genes had an effect on the growth of $P$. microspora NK17. The diameter of fungi on PLA plate was recorded every day for 3 days, and the dry weight of mycelia in PLB broth was measured after 3 days and 7 days, respectively.

\section{High Performance Liquid Chromatography (HPLC) Profiling for Secondary Metabolites}

Conidia suspension in $3 \mathrm{ml}$ sterile $\mathrm{ddH}_{2} \mathrm{O}$ containing approximately $10^{5}$ conidia was added to $3 \mathrm{~g}$ sweet potato waste medium (in $50 \mathrm{ml}$ flask). Grown at $28^{\circ} \mathrm{C}$ for 13 days, $20 \mathrm{ml}$ methanol was added into the flake to extract metabolites, at room temperature for $24 \mathrm{~h}$. The organic phase was filtrated, and $25 \mu \mathrm{l}$ was subjected to HPLC analysis. The HPLC process followed the protocols described by Wang et al. [20].

\section{Results}

\section{Structural Characterization of Secondary Metabolites in P. microspora}

In a previous report, we characterized the secondary metabolite pestalotiollide $\mathrm{B}$ in the culture of $P$. microspora (Compound 1) [9]. To illustrate the chemical structure of more secondary products of the fungus, we chose four other metabolites that have the highest peaks on HPLC spectrum (Figs. 1 and S1). Their structures were determined via similar biochemical approaches used for the structure study of compound $\mathbf{1}[9,14]$, which were presented here, namely, pestalotiollide $C(2), 1^{\prime}, 2^{\prime}$-dehydropenicillide (3), 3'-methoxy-1'2'-dehydropenicillide (4) and 1',2'-epoxy3',4'-didehydropenicillide (5) (Figs. 1 and S1). The structure data of these compounds suggest that dibenzodioxocinone derivatives are the major products in P. microspora NK17, under the growth conditions.

Compound 2 was a yellow amorphous powder when purified and its molecular formula was determined as $\mathrm{C}_{21} \mathrm{H}_{22} \mathrm{O}_{7}$ according to HRMS data $\left(\mathrm{m} / \mathrm{z}=409.1271[\mathrm{M}+\mathrm{Na}]^{+}\right.$, $\left.795.2630[2 \mathrm{M}+\mathrm{Na}]^{+}\right)$, indicating 2 contained an unsaturated bond at site 11 (Fig. S2b). The ${ }^{1} \mathrm{H}-\mathrm{NMR}$ data showed that 2 possessed two methyl groups and methoxyl (Fig. S2j and Table S2). The results of ${ }^{13} \mathrm{C}-\mathrm{NMR}$ and ${ }^{1} \mathrm{H}-\mathrm{NMR}$ spectra suggested that there were two benzenoid rings, one carbonyl carbon and one carbon-carbon double bond at end group (Figs. S2j, S2k, and Table S3), just like compound 1 (Figs. S2g and S2h). Combined with HSQC data (Figs. S2i and S2l), the structure of 2 shared the same molecular framework with pestalotiollide B (1). Interestingly, 1 and 2 have the same molecular formula and shared similar spectra of ${ }^{1} \mathrm{H}-\mathrm{NMR}$ and ${ }^{13} \mathrm{C}-\mathrm{NMR}$ (Figs. S2a and S2b, Tables S2 and S3). To distinguish their structure, the circular dichroism (CD) spectrum was conducted and the difference was observed at the 2 and pestalotiollide B [9]. As shown in Fig. S2c, compound 2 showed a positive Cotton effect $(\Delta \varepsilon=$ $+13.42,255 \mathrm{~nm})$ and a negative Cotton effect $(\Delta \varepsilon=-19.32$, $285 \mathrm{~nm})$, which was similar to the compound (1R, 2S)-4 determined by Ishida, who determined all of the four configurations of 1-phenylpropane-1,2-diols by comparing their CD spectra [21]. Via this data, the conformation of compound 2 was characterized to be $\left(1^{\prime} R, 2^{\prime} S\right.$ ) (Figs. $1 B$ and $\mathrm{S} 2 \mathrm{~b})$.

Compound 3 was lyophilized to white powder. The data of HRMS at $m / z$ 393.1319[M+Na] $]^{+}$and $m / z 763.2719[2 \mathrm{M}+\mathrm{Na}]^{+}$ suggested a molecular formula $\mathrm{C}_{21} \mathrm{H}_{22} \mathrm{O}_{6}$ (Fig. S2d). Also, this product had a similar NMR spectrum to 1 , suggesting $\mathbf{3}$ was a dibenzodioxocinone derivative (Figs. S2m and S2n, 
Tables S2 and S3). Correlation in HMBC from an olefinic methine proton $\mathrm{H}-1^{\prime}\left(\delta_{H}=6.82 \mathrm{ppm}, 1 \mathrm{H}, J=16.24 \mathrm{~Hz}\right)$, which was coupled to an olefinic methine proton $\mathrm{H}-2^{\prime}$ $\left(\delta_{H}=6.4 \mathrm{ppm}, 1 \mathrm{H}, J=16.24 \mathrm{~Hz}\right)$ in ${ }^{1} \mathrm{H}-\mathrm{NMR}$, to $\mathrm{C}-2, \mathrm{C}-3$ and $\mathrm{C}-4$, established a carbon-carbon bond between $1^{\prime}$ and $2^{\prime}$ instead of two hydroxyls (Figs. 1A and S2p). A long-range ${ }^{1} \mathrm{H}_{-}{ }^{13} \mathrm{C}$ in coupling of the two methyl protons $\mathrm{H}_{3}-4^{\prime}, 5^{\prime}$ $\left(\delta_{H}=1.46 \mathrm{ppm}, 3 \mathrm{H}\right)$ to a quaternary carbon C-3' $\left(\delta_{C}=71.19\right.$ ppm) and C-2' $\left(\delta_{C}=140.6 \mathrm{ppm}\right)$ was observed. The HMBC data together suggest the structure of 3 (Figs. 1 and S2d).

Likewise, HRMS spectra of 4 gave rise to a molecular formula of $\mathrm{C}_{22} \mathrm{H}_{24} \mathrm{O}_{6}$ (obsd: $407.1461 \mathrm{~m} / \mathrm{z}[\mathrm{M}+\mathrm{Na}]^{+}$, Fig. S2e). ${ }^{1} \mathrm{H}-\mathrm{NMR}$ and ${ }^{13} \mathrm{C}-\mathrm{NMR}$ data of 4 suggested a similar structure to 3 except the extra signals of the $-\mathrm{OCH}_{3}$ substituent (Figs. S2q and S2r, Tables S2 and S3), which were absent in 3 (Figs. S2m and S2n). Long-range ${ }^{1} \mathrm{H}^{13}{ }^{13} \mathrm{C}$ couplings from $-\mathrm{OCH}_{3}$ to $3^{\prime}$ established a $3^{\prime}$-methoxy- $\Delta^{12^{\prime}}$ side chain of 4 (Figs. 1A, S2e, and S2t).

Compound 5 formed faint yellow powder. HRMS data exhibited an ion peak at $\mathrm{m} / \mathrm{z} 391.1158[\mathrm{M}+\mathrm{Na}]^{+}$, corresponding to the molecular formula $\mathrm{C}_{21} \mathrm{H}_{20} \mathrm{O}_{6}$ (Fig. S2f). The ${ }^{1} \mathrm{H}$ - and ${ }^{13} \mathrm{C}$ - NMR spectra of 5 displayed similar chemical shifts with compound 1 except for a minor difference (Figs. S2u and S2v, Tables S2 and S3). Corresponding to the molecular formula, 5 should possess a $1^{\prime}, 2^{\prime}$-epoxide consistent. Based on the HSQC data, the carbons located at C-1' and C-2' should be methine (Figs. 1B, S2f, and S2w).

\section{Identification of a Gene Cluster Responsible for the Biosynthesis of Dibenzodioxocinones}

From the structure of the five compounds, we confirmed that the carbon backbones of dibenzodioxocinones were biosynthesized by PKS. From the production of pestalotiollide B by 41 PKS-deleted strain, PKS8 produced little [17], suggesting that $p k s 8$ plays the major part in the biosynthesis of pestalotiollide B. Structural analysis of PKS8 revealed that this protein contained 5 putative functional domains, starter unit: ACP transacylase (SAT) domain, ketoacyl synthase (KS) domain, PKS-AT (AT) domain, iterative type I PKS product template (PT) domain, and acyl carrier protein (ACP) domain (Fig. 2A). To explore the genetic foundation underlying the biosynthesis of dibenzodioxocinones in P. microspora, we took an approach via differential profiling by RNA-seq between the mutant strain $g \alpha 1 \Delta$, which did not produce dibenzodioxocinones due to the loss of $G$ protein $\alpha$ subunit, and the wild type (Fig. 2B) [18]. A gene cluster containing 21 genes, including studied gene pks8 (GME11356) [17], was found to have an obviously lower expression level in RNA-seq, by $76 \%$ to
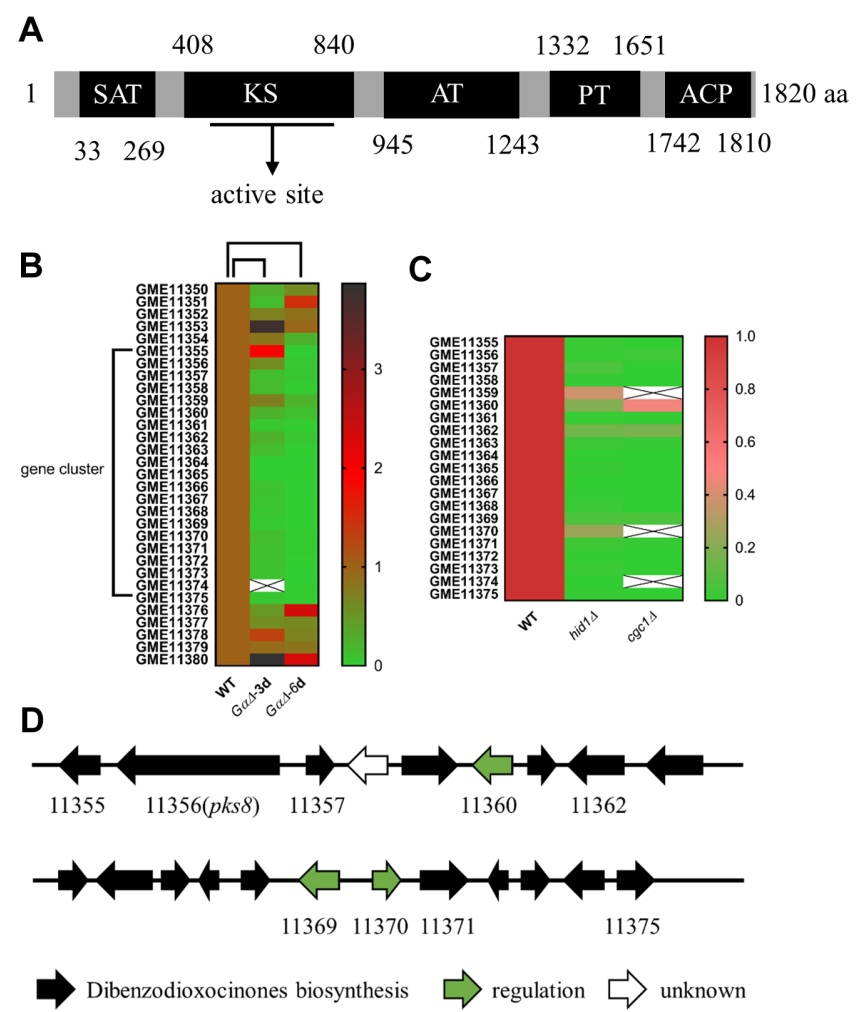

Fig. 2. Functional domains of PKS8 and RNA-seq analysis to identify the gene cluster of dibenzodioxocinone in NK17.

(A) Architecture of PKS8 conserved domains. Domains include SAT, $\mathrm{KS}, \mathrm{AT}, \mathrm{PT}$ and ACP. The locations of domains and the active site are shown. (B) Gene expression of GME11350 to GME11380 in pga1 $1 \Delta$ and wild-type strain after culturing for 3 and 6 days, respectively. The quantity of the wild type was standardization to ' 1 ' in both 3 and 6 days' data. (C) Gene expression of GME11355 to GME11375 in hid1 and $\operatorname{cgc} 1 \Delta$ strains after culturing for 6 days. The gene expression quantity of wild type was standardization to ' 1 '. (D) The gene cluster containing 21 ORFs (see Table 1). WT, wild type.

$100 \%$, for most of the genes in the mutant, e.g. from GME11355 to GME11375 (Figs. 2B, 2C, and 2D). Of the 21, 5 genes were deduced to participate in the generation of the backbone structure, i.e. GME11356, GME11357, GME11358, GME11365 and GME11367, as well as three regulatory genes, GME11360, GME11369 and GME11370 showed high similarity with genes in diphenyl ether gene cluster in Pestalotiopsis fici [22]. In addition, a prediction by AntiSMASH suggested that these 21 genes were located in a probable gene cluster and were likely expressed in a cooperative way (Fig. 2D).

Demonstration of the Role of the Gene Cluster in Dibenzodioxocinone Biosynthesis

In previous studies, Chen et al. constructed a $p k s 8$-deleted 
Table 1. Genes in a dibenzodioxocinone biosynthesis cluster and putative conserver domain.

\begin{tabular}{|c|c|c|c|c|c|}
\hline Gene & Size (bp/aa) & Blastp Homologue & Identity (\%) & Conserver domain & E value \\
\hline GME11355 & $807 / 269$ & Coccidioides immitis, $\mathrm{CtnB}$ & 55 & Serine hydrolase (FSH1) & $7 \mathrm{e}-100$ \\
\hline GME11356 & $5628 / 1876$ & Diaporthe helianthi, PKS & 72 & SAT-KS-AT-PT-ACP & 0 \\
\hline GME11357 & $1041 / 347$ & Diaporthe helianthi, metallo-beta-lactamase protein & 63 & Metallo-beta-lactamase & $1.05 \mathrm{e}-26$ \\
\hline GME11358 & $1350 / 450$ & Aspergillus mulundensis, question oxidase & 55 & Protein of unknown function & $5.59 \mathrm{e}-122$ \\
\hline GME11359 & $1161 / 387$ & Eutypa lata, short-chain dehydrogenase reductase protein & 59 & DH (dehydrogenase) domain & $2.75 \mathrm{e}-24$ \\
\hline GME11360 & $1896 / 632$ & Clooetotrichum orbiculare, C6 transcription factor & 36 & Transcription factor domain & $7.58 \mathrm{e}-44$ \\
\hline GME11361 & $855 / 285$ & Diaporthe helianthi, short-chain dehydrogenease & 69 & DH domain & $6.53 e-65$ \\
\hline GME11362 & $1773 / 591$ & Diaporthe helianthi, choline dehydrogenase & 69 & DH domain & $1.41 \mathrm{e}-84$ \\
\hline GME11363 & $1923 / 641$ & Aspergillus novofumigatus, cytochrome P450 & 52 & Monooxygenase domian & $1.62 \mathrm{e}-15$ \\
\hline GME11364 & $444 / 148$ & $\begin{array}{l}\text { Pochonia chlamydosporia, monooxygenase } \\
\text { domain-containing protein }\end{array}$ & 54 & Monooxygenase domian & $5.7 \mathrm{e}-5$ \\
\hline GME11365 & $2133 / 711$ & Penicillium occitanis, multicopper oxidase & 76 & Oxidoreductase domain & $1.89 \mathrm{e}-65$ \\
\hline GME11366 & $1419 / 473$ & Talaromyces cellulolyticus, O-methyltransferase & 68 & O-methyltransferase domain & $1.88 \mathrm{e}-20$ \\
\hline GME11367 & $789 / 263$ & Hypoxylon sp., putative NAD-dependent dehydratase & 64 & NAD-binding domain & $9.57 \mathrm{e}-24$ \\
\hline GME11368 & $1212 / 404$ & Aspergillus taichungensis, aryl-alcohol dehydrogenase & 71 & $\begin{array}{l}\text { Predicted oxidoreductase } \\
\text { domain }\end{array}$ & $2.74 \mathrm{e}-90$ \\
\hline GME11369 & $1479 / 493$ & Talaromyces mameffei ATCC, O-mathyltransferase & 39 & $\begin{array}{l}\text { No putative conserved } \\
\text { domains have been detected }\end{array}$ & - \\
\hline GME11370 & $1230 / 410$ & Rasamsonia emersonii CBS, C6 transcription factor (AfIR) & 33 & $\begin{array}{l}\text { Aflatoxin regulation protein } \\
\text { domain }\end{array}$ & $4.75 \mathrm{e}-28$ \\
\hline GME11371 & $1881 / 627$ & Phialocephata scopiformis, MFS general substrate transporter & 50 & Putative transporter domain & $2.58 \mathrm{e}-18$ \\
\hline GME11372 & $606 / 202$ & Diaporthe helianthi, scytalone dehydratase & 81 & Scytalone dehydratase domain & $4.99 \mathrm{e}-75$ \\
\hline GME11373 & $933 / 311$ & Diaporthe helianthi, tetrahydroxynaphthalene reductase-1 & 87 & $\begin{array}{l}\text { Tetrahydroxynaphthalene } \\
\text { reductase-like domain }\end{array}$ & $1.67 \mathrm{e}-108$ \\
\hline GME11374 & $1797 / 599$ & Macrophomina phaseolina, AMP-dependent synthetase & 51 & AMP-binding domain & $1.39 \mathrm{e}-38$ \\
\hline GME11375 & $1455 / 485$ & Diaporthe helianthi, aromatic prenyltransferase(PTase) & 70 & PTases domain & $1.11 \mathrm{e}-129$ \\
\hline
\end{tabular}

strain and found that the production of PB was decreased by $95 \%$ [17]. To further demonstrate the status of the $p k s 8$ gene cluster in the biosynthesis of dibenzodioxocinones, all 21 genes (GenBank Accession Nos. are MK590975, APX43979.1, and MK590976-MK590994 for GME11355, GME11356, and GME11357-GME11375, respectively) in this cluster were analyzed by protein BLAST to verify their functions (Table 1). From the results of BLAST, pks8-cluster contains one PKS gene (GME11356), two transcription factor genes (GME11360 and GME11370), one transport protein (GME11371), and seventeen structure-related genes. Finally, six genes, GME11355 (a predicted salicylate hydroxylase encoding gene), GME11357 (a predicted hydrolase encoding gene), GME11360, GME11362 (a predicted oxidoreductase activity protein), GME11371 and GME11375 (encodes an aromatic prenyltransferase activity relative protein) were chosen for knockout to verify their role in the biosynthesis of dibenzodioxocinones (Table 1).
These six genes contained all types of genes in $p k s 8$-cluster, which may be helpful to declare the function of this cluster in the biosynthesis pathway of dibenzodioxocinones. Additionally, GME11376, a predicted secretory lipaseencoding gene, was disrupted to determine the boundary of the cluster. All of the seven genes were successfully disrupted one by one by homologous targeting via ATMT in orotidine $5^{\prime}$-phosphate decarboxylase (ODCase) deficient strain, ura3s-1 (from NK17) [17] and confirmed by Southern blotting (Figs. 3A, 3B, and S3). The secondary metabolites in the resulting mutants were profiled by HPLC. Compound $\mathbf{5}$ was also monitored to evaluate the production of dibenzodioxocinones in the strains, as the peak of compound $\mathbf{5}$ was isolated from other peaks in HPLC spectrum (Fig. S4). As the results showed, deletion of GME11355, GME11360 and GME11375 completely blocked the production of dibenzodioxocinones, whereas knockout of either GME11362 or GME11371 significantly 
A
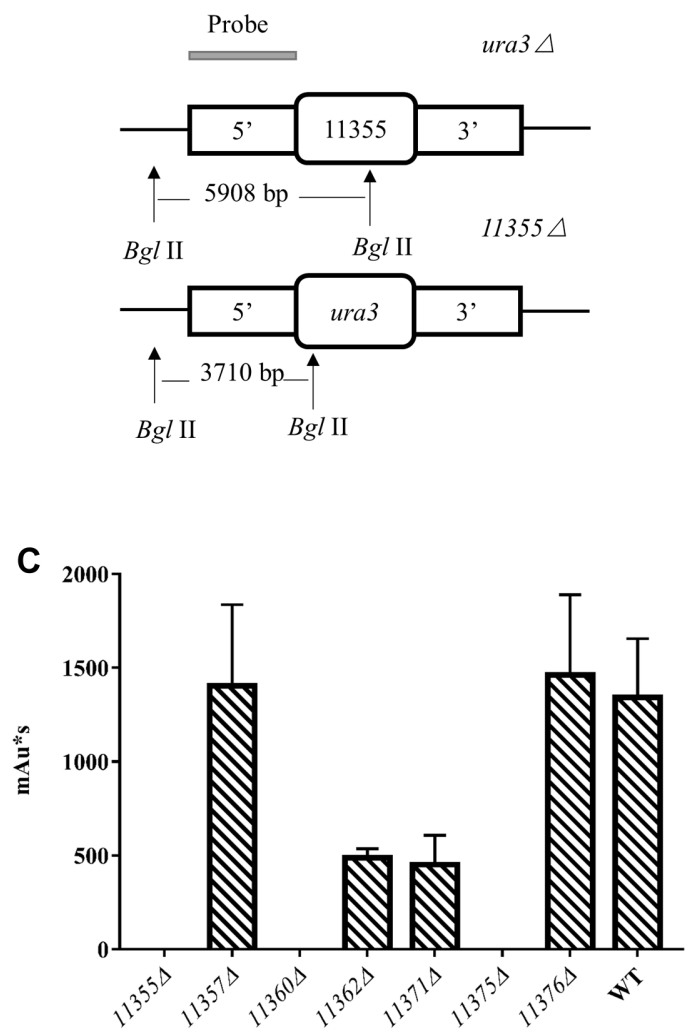

B

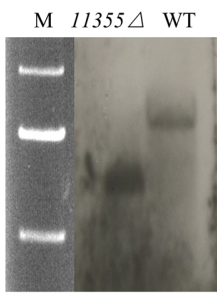

M $11357 \triangle$ WT

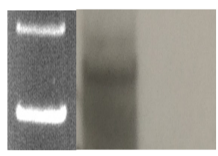

M $11360 \triangle$ WT

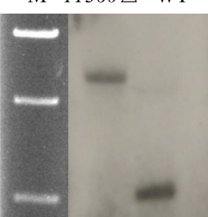

M $11362 \triangle \mathrm{WT}$

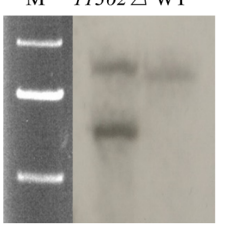

M 11371 $\triangle$ WT

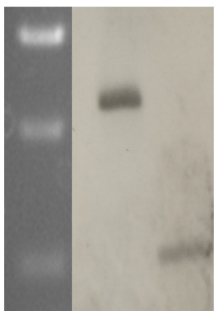

M $11375 \triangle \mathrm{WT}$

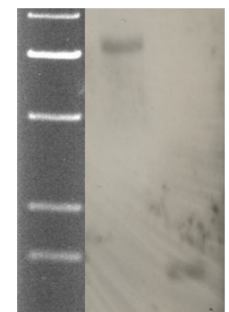

M $11376 \triangle$ WT

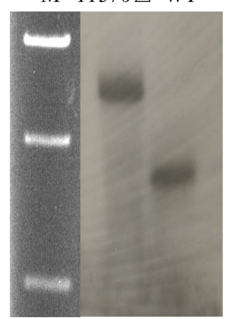

Fig. 3. Disruption of target genes in the defined cluster.

(A) Diagram for disruption of GME11355 Southern blotting. GME11355 was deleted by homologous recombination in ura3 4 mutation strain. $5^{\prime}, 5^{\prime}-$

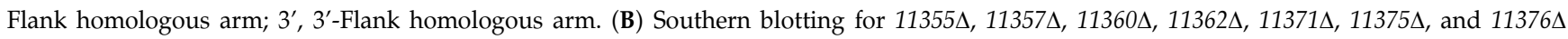

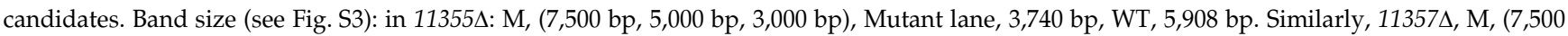
bp, 5,000 bp), mutant 6,749 bp, WT, no band; 11360د, M, (5,000 bp, 3,000 bp, 1,500 bp), mutant, 3,742 bp, WT, 1,512 bp; $11362 \Delta, \mathrm{M},(7,500$ bp. 5,000 bp. 3,000 bp), mutant, 6,603 bp and 3,437 bp, WT, 6,418 bp; 11371 , M, (3,000 bp, 1,500 bp, 1,000 bp), mutant, 2,382 bp, WT, 1,009 bp; 11375 , M, (7,500 bp, 5,000 bp, 3,000 bp, 1,500 bp, 1,000 bp), mutant, 5,599 bp, WT, 792 bp; 11376 , M, (5,000 bp, 3,000 bp, 1,500 bp), mutant, 4,368 bp, WT, 2,392 bp. M: marker, WT, wild type. (C) The peak area of compound 5 on HPLC was calculated for the mutant strains in sweet potato waste fermentation, for 13 days at $28^{\circ} \mathrm{C}$. The production of compound 5 was abolished in $11355 \Delta, 11360 \Delta$ and $11375 \Delta$ strains, and was decreased by approximately $67 \%$ in $11362 \Delta$ and $11371 \Delta$ strains, while it did not obviously change in the other two strains.

decreased dibenzodioxocinones by approximately $67 \%$. Rather, deletion of GME11376 slightly increased the production of compound 5 by $7 \%$ (Figs. 3C and S4). These data strongly demonstrate these candidate genes play a critical role in the biosynthesis of dibenzodioxocinones.

\section{Phenotypic Consequence of the Disruption of the Dibenzodioxocinone Genes on Growth}

To investigate whether these candidate genes had an additional effect on the growth of $P$. microspora, we observed the growth of NK17 and the mutants both on agar and in liquid broth. Two of the mutant strains, $11360 \Delta$ and 11362 , formed significantly smaller colonies on PLA plate than the wild-type colony. The diameter of the strains decreased by approximately $29 \%$ and $13 \%$, respectively, compared to the wild type, after 2 days' incubation, or $21 \%$ and $13 \%$ after 3 days (Fig. $4 \mathrm{~A}$ ), suggesting that only GME11360 and GME11362, encoding a transcription factor and an oxidoreductase, were involved in the growth of $P$. microspora in PLA medium. For the other mutant strains, no extinguishable difference in growth was observed. Furthermore, if cultured in PLB, all these mutated genes showed little effect on the growth of NK17 (Fig. 4B).

\section{Discussion}

Filamentous fungi are well recognized for their strong capacity to produce an abundance of natural products, 


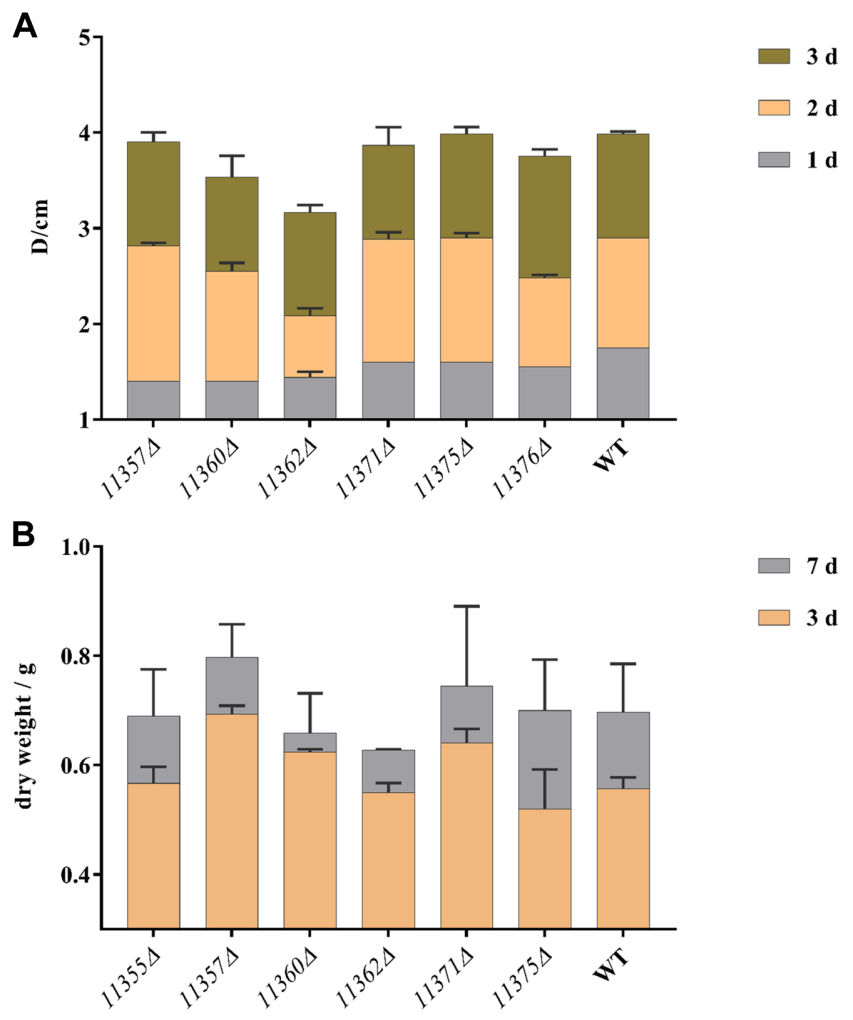

Fig. 4. Effect of disruption of the genes on the growth of P. microspora NK17.

(A) The colony size by diameter on PLA plates was determined. (B) The dry weight of mycelium of fungal strains at 3 days and 7 days in PLB, respectively.

such as terpenoids $[7,16]$ and polyketides $[2,5]$. In a former article, we reported the identification of the first dibenzodioxocinone in P. microspora NK17, pestalitiollide $\mathrm{B}$, which is notably present at a high yield in fungus [9]. We continue our mission with further characterization of more secondary metabolites in this fungus in order to virtually decipher the secondary metabolic pathways. Four more products, in addition to pestalitiollide $B$, were structurally determined in this study and altogether, these five molecules that appear on HPLC spectrum as the five highest peaks (except the melanin peaks) were all involuntarily dibenzodioxocinones with variable side chains (Figs. 1 and S2). Thus, dibenzodioxocinones are the dominant secondary metabolites in P. microspora, under the current growth condition. Significantly, we identified a gene cluster in this fungus and showed its role in the biosynthesis of pestalotiollide B and the other dibenzodioxocinones. This is fortunately accomplished by utilizing the differential gene expression information (RNA-seq) between the wild-
Table 2. Genetic organization comparison of $p k s 8$ cluster, $p t a$ cluster and $m d p$ cluster.

\begin{tabular}{ccccc}
\hline $\begin{array}{c}p k s 8 \text { cluster } \\
\text { genes }\end{array}$ & $\begin{array}{c}p t a \text { cluster } \\
\text { genes }\end{array}$ & $\begin{array}{c}\text { Identity } \\
(\%)\end{array}$ & $\begin{array}{c}m d p \text { cluster } \\
\text { genes }\end{array}$ & $\begin{array}{c}\text { Identity } \\
(\%)\end{array}$ \\
\hline GME11356 & $p t a A$ & 63.09 & $m d p G$ & 65.82 \\
GME11357 & $p t a B$ & 57.1 & - & - \\
GME11358 & $p t a J$ & 45.09 & $m d p L$ & 55.7 \\
GME11365 & $p t a E$ & 46.06 & - & - \\
GME11367 & - & - & $m d p K$ & 62.88 \\
GME11369 & - & - & $m d p A$ & 39.21 \\
GME11370 & $p t a R 2$ & 30.13 & - & - \\
GME11373 & - & - & $m d p C$ & 75.67 \\
GME11374 & - & - & $m d p I$ & 39.32 \\
\hline
\end{tabular}

type strain and a mutant strain $g a 1 \Delta$ in which the $G$ protein $\alpha$ subunit encoding gene was mutated, as ga1s lost the capacity to produce dibenzodioxocinones [18]. By analyzing the RNA-seq data of $g a 1 \Delta$, we found the gene cluster that contains 21 genes, numbered from GME11355 to GME11375, including pks8 (GME11356), which is a polyketide synthase gene for the biosynthesis of pestalotiollide B (Fig. 2A) [17]. Considering the loss of dibenzodioxocinones in the disruption mutant $p k s 8 \Delta$, the existence of $p k s 8$-dependent biosynthesis of the metabolites was highly desirable. One of the boundaries of this cluster was confirmed by deleting GME11375 and GME11376, respectively (Figs. 3B and 3C). In the mutant strain $11375 \Delta$, dibenzodioxocinones were hardly detectable, while in $11376 \Delta$ little change was detected in the production of dibenzodioxocinones or the growth of NK17. We meanwhile attested by homologous targeting several genes within the cluster pending a role in the biosynthesis of dibenzodioxocinones, namely, three structural genes, GME11355, GME11360, and GME11375, and two regulatory ones, GME11362 and GME11371. Notably, blasting against the protein sequence of $p k s 8$ generated a high homology to ptaA (Table 2), a PKS gene involving in the biosynthesis of pestheic acid in a sibling species P. fici [22], and $m d p G$ (Table 2), an essential PKS gene of $m d p$ gene cluster in the biosynthesis of emodin and its derivatives [23]. Pestheic acid has a resembling diphenyl ether structure with dibenzodioxocinones, suggesting that they may share a similar PKS-dependent biosynthesis pathway. However, when the $\beta$-lactamase (GME11357) was disrupted, the production of dibenzodioxocinones rarely decreased. $\beta$-lactamase was reported to be a novel type of thioesterase responsible for release of products in polyketide biosynthesis such as endocrocin or emodin 
acetyl-CoA<smiles>CC1=CC(=O)C2C(=O)c3c(O)cc(O)cc3C(=O)C2C(O)=C1C(=O)O</smiles><smiles>Cc1cc(O)c2c(c1)C(=O)c1cc(O)cc(O)c1C2=O</smiles>

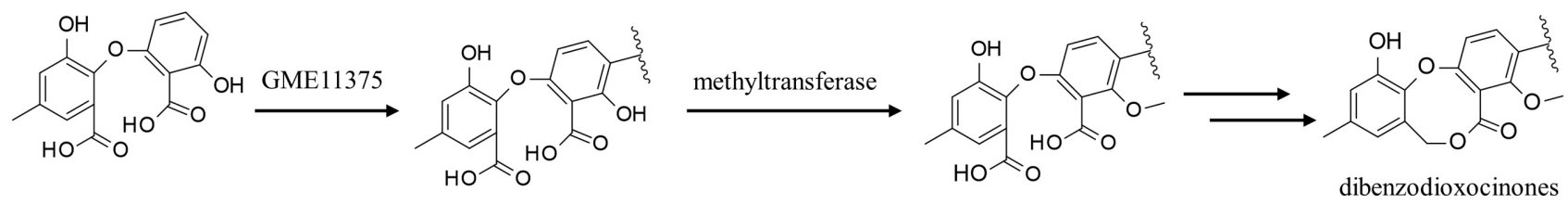

Fig. 5. Proposed biosynthesis pathway leading to formation of the carbon bone of dibenzodioxocinones in P. microspora. The genes in the pathway were indicated.

(Figs. 1B and 5) [24]. Via protein functional BLAST in genome of NK17 we found out at least 23 proteins containing $\beta$-lactamase domain, revealing that the function of GME11357 may be compensated by other $\beta$-lactamases in $11357 \Delta$ strains.

Based on the results above, and some compounds reported earlier from other fungi, including atrochrysone carboxylic acid [22], endocrocin anthrone, endocrocin [23], tenellic acid B and monodictyphenone [23], the biochemical steps toward dibenzodioxocinones were proposed here for P. microspora (Fig. 5). The molecules of dibenzodioxocinones share an analogous diphenyl ether structure with pesthetic acid, indicating that the synthesis initiates from acetate or malonate units, and is then catalyzed by PKS8 (GME11356). While PKS and $\beta$-lactamase were coincubated in the presence of malonyl-CoA, all of atrochrysone carboxylic acid, endocrocin, endocrocin anthrone and emodin could be detected according to the results of in vitro reactions by Awakawa [24]. Thus, it was likely that PKS8 catalyzed the closure of two benzene rings, $\mathrm{A}$ and $\mathrm{B}$, of atrochrysone carboxylic acid (Fig. 5), and then this product was released from PKS8 by GME11357 or other $\beta$-lactamases in NK17. By conserved domain analysis we noticed that PKS8 lacked the thioesterase (TE) domain [24], which belonged to a hydrolase superfamily and was important to the cyclizing of the third ring of atrochrysone carboxylic acid [25] (Fig. 5), compared with other PKS reported before. In our data, disrupting of putative serine hydrolase (GME11355) resulted in the disappearance of dibenzodioxocinones, so we speculated that GME11355 played the role of TE and catalyzed the cyclization reaction of the $C$ ring (Fig. 5). By comparing the genetic organization between $p k s 8$ and $m d p$ cluster, three genes in pks8 cluster, GME11358, GME11367 and GME11373, showed high protein similarity to the genes involved in the catalytic reaction from emodin to monodictyphenone in $m d p$ cluster (Table 2) [23]. It is thus possible that emodin in NK17 was further oxidized by several genes, such as GME11358, GME11367, GME11373 and the other genes [26], to modify the $A$ and $C$ rings and open the B ring, and finally transformed into monodictyphenone (Fig. 5). GME11365 was putative to be a multicopper oxidase, which had the same conserved domain with DHGO, which catalyzed the stereospecific phenol oxidative coupling in dihydrogeodin in A. terreus [27], and PtaE, which was conjectured to convert chloroisosulochrin to a spiran pestheic acid in P. fici [22]. Besides, protein BLAST results showed that GME11365 has a $46 \%$ identity with PtaE (Table 2), suggesting that 
GME11365 was involved in a similar oxidative coupling reaction [22] as shown in Fig. 5.

Prenyltransferase was reported to be responsible for the prenylation of flavonoids in Sophora flavescens [28, 29]. A functional similar gene, GME11375, was found in $p k s 8$ cluster, and its disruption mutation rarely produced dibenzodioxocinones, suggesting that GME11375 may catalyze the addition reaction between the $\mathrm{C} 5$ side chains and the carbon bone of dibenzodioxocinones. However, the intermediates without side chain (compound A in Fig. S5a) was not detected in the culture of $11375 \Delta$ by HPLC (Fig. S4) and LC-MS (Figs. S5b and S5c). As shown in Fig. S5, if the reaction catalyzed by GME11375 occurred at the last step of dibenzodioxocinones biosynthesis, compound A (Fig. S5a) should be produced, and the ion peaks at $287.0920[\mathrm{M}+\mathrm{H}]^{+}$ or $309.0739[\mathrm{M}+\mathrm{Na}]^{+}$should be observed in LC-MS spectrum (Figs. S5b and S5c). However, we can not find these ion peaks, possibly because the addition of side chain was indispensable in some other modification reactions in the biosynthesis pathway of dibenzodioxocinones, and the unstable intermediates were quickly converted. The remaining biochemical reactions to the final product dibenzodioxocinones should be methylation catalyzed by methyltransferase GME11366 or GME11369, and reduction and lactonization reaction catalyzed by a series of oxidordeuctases (Fig. 5).

In conclusion, we determined the structure of four major secondary metabolites in the culture of $P$. microspora. They are the analogs of CEPT inhibitors (dibenzodioxocinones). The gene cluster was defined in the genome that deductively encodes most of the enzymes for the chemical reactions. Amid the genes within the cluster, $p k s 8$ plays the major role in the biosynthesis of dibenzodioxocinones. The biochemical steps leading to the formation of the core structure of dibenzodioxocinones were proposed. This work sheds light on the genetic and biochemical basis for the biosynthesis of dibenzodioxocinones in fungi, and shall help to create new CEPT-inhibiting derivatives for novel drugs.

\section{Acknowledgments}

This study was funded by the National Science Foundation of China (81871629 and continually 31470251).

\section{Conflict of Interest}

The authors have no financial conflicts of interest to declare.

\section{References}

1. Kawahara $T$, Itoh M, Izumikawa $M$, Sakata N, Tsuchida $T$, Shin-ya K. 2013. Three eremophilane derivatives, MBJ-0011, MBJ-0012 and MBJ-0013, from an endophytic fungus Apiognomonia sp. f24023. J. Antibiot. 66: 299-302.

2. Lin ZJ, Zhu TJ, Fang YC, Gu QQ, Zhu WM. 2008. Polyketides from Penicillium sp. JP-1, an endophytic fungus associated with the mangrove plant Aegiceras corniculatum. Phytochemistry 69: 1273-1278.

3. Challinor VL, Bode HB. 2015. Bioactive natural products from novel microbial sources. Ann. NY Acad. Sci. 1354: 8297.

4. Hu Y, Zhou YX, Mao ZJ, Li HH, Chen FS, Shao YC. 2017. $\mathrm{NAD}^{+}$-dependent HDAC inhibitor stimulates Monascus pigment production but inhibit citrinin. AMB Express 7: 116.

5. Xu J, Kjer J, Sendker J, Wray V, Guan HS, Edrada RA, et al. 2009. Chromones from the endophytic fungus Pestalotiopsis $\mathrm{sp}$. Isolated from the Chinese mangrove plant Rhizophora mucronata. J. Nat. Prod. 72: 662-665.

6. David AG, Jonathan G, Alex M, Ana R, Inaki RT, Roger ES. 2016. Sterol and genomic analyses validate the sponge biomaker hypothesis. PNAS 113: 2684-2689.

7. Edilu A, Adane L, Woyessa D. 2015. In vitro antibacterial activities of compounds isolated from roots of Caylusea abyssinica. Ann. Clin. Microbiol. Antimicrob. 14: 15.

8. Brückner D, Hafner FT, Li V, Schmeck C, Telser J, Vakalopoulos A, et al. 2005. Dibenzodioxocinones-A new class of CETP inhibitors. Bioorg. Med. Chem. Lett. 15: 36113614.

9. Niu XL, Hao XR, Hong ZY, Chen LF, Yu X, Zhu XD. 2015. A putative histone deacetylase modulates the biosynthesis of Pestalotiollide B and conidiation in Pestalotiopsis microspora. J. Microbiol. Biotechnol. 25: 579-588.

10. Liu L, Li Y, Cao Y, Guo LD, Liu G, Che YS. 2013. Spiroketals of Pestalotiopsis fici provide evidence for a biosynthetic hypothesis involing diversified Diels-Alder reaction cascades. J. Org. Chem. 78: 2992-3000.

11. Yang XL, Awakawa T, Wakimoto T, Abe I. 2013. Induced production of novel prenyldepside and coumarins in endophytic fungi Pestalotiopsis acaciae. Tetrahedron Lett. 54: 5814-5817.

12. Kawamura H, Kaneko T, Koshino H, Esumi Y, Uzawa J, Sugawara F. 2000. Penicillides from Penicillium sp. isolated from Taxus cuspidata. Nat. Prod. Lett. 14: 477-484.

13. Kuroda K, Morishita Y, Saito Y, Ikuina Y, Ando K, Kawamoto I, et al. 1994. AS-186 compounds, new inhibitors of acyl-CoA : cholesterol acyltransferase from Penicillium asperosporum KYI 635. J. Antibiot. 47: 16-22.

14. Zhang Y, Li XM, Shang Z, Li CS, Ji NY, Wang BG. 2012. Meroterpenoid and diphenyl ether derivatives from Penicillium sp. MA-37, a fungus isolated from marine mangrove rhizospheric soil. J. Nat. Prod. 75: 1888-1895. 
15. Bi JN, Ji Y, Pga1n J, Yu Y, Chen H, Zhu XD. 2011. A new taxol-producing fungus (Pestalotiopsis malicola) and evidence for taxol as a transient product in the culture. Afr. J. Biotechnol. 10: 6647-6654.

16. Strbel G, Yang XS, Sears J, Kramer R, Sidhu RS, Hess W.M. 1996. Taxol from Pestalotiopsis microspora, an endophytic fungus of Taxus wallachiana. Microbiol. 142: 435-440.

17. Chen LF, Li YY, Zhang Q, Wang D, Akhberdi O, Wei DS, et al. 2017. Improved pestalotiollide B production by deleting competing polyketide synthase genes in Pestalotiopsis microspora. J. Ind. Microbiol. Biotechol. 44: 237-246.

18. Yu X, Liu H, Niu XL, Akhberdi O, Wei DS, Wang D, et al. 2017. The Ga1-cAMP signaling pathway controls conidiation, development and secondary metabolism in the taxolproducing fungus Pestalotiopsis microspora. Microbiol. Res. 203: 29-39.

19. Yu X, Huo L, Liu H, Chen LF, Wang Y, Zhu XD. 2015. Melanin is required for the formantion of the multi-cellular conidia in the endophytic fungus Pestalotiopsis microspora. Microbiol. Res. 179: 1-11.

20. Wang D, Akhberdi O, Hao XR, Chen LF, Liu YJ, Zhu XD. 2017. Amino acid sensor kinase Gen2 is required for conidiation, secondary metabolism, and cell wall integrity in the Taxol-producer Pestalotiopsis microspora. Front Microbiol. 8: 1879.

21. Ishida T, Bounds SVJ, Caldwell J, Drake A, Takeshita M. 1996. The absolute configuration of the four stereoisomers of trans-anethole diol (1-(4"'-Methoxyphenyl)-1,2-propanediol), a metabolite of anethole in the rat. Tetrahedron Asymmetry 7: 3113-3118

22. Xu XX, Liu L, Zhang F, Wang WZ, Li JY, Guo LD, et al. 2014. Identification of the first diphenyl ether gene cluster for pestheic acid biosynthesis in plant endophyte Pestalotiopsis fici. Chembiochem 15: 284-292.

23. Chiang YM, Szewczyk E, Davidson AD, Entwistle R, Keller NP, Wang CCC, et al. 2010. Characterization of the Aspergillus nidulans Monodictyphenone Gene Cluster. Appl. Environ. Microbiol. 76: 2067-2074.

24. Awakawa T, Yokota K, Funa N, Doi F, Mori N, Watanabe H, et al. 2009. Physically discrete $\beta$-lactamase-type thioesterase catalyzes product release in atrochrysone synthesis by iterative type I polyketide synthase. Chem. Biol. 16: 613-623.

25. Crawford JM, Thomas PM, Scheerer JR, Vagstad AL, Kelleher NL, Townsend CA. 2008. Deconstruction of iterative multidomain polyketide synthase function. Science 320: 243246.

26. Cary JW, Ehrlich KC, Bland JM, Montalbano BG. 2006. The aflatoxin biosynthesis cluster gene, aflX, encodes an oxidoreductase involved in conversion of versicolorin A to demethylsterigmatocystin. Appl. Environ. Microbiol. 72: 10961101.

27. Fujii I, Iijima H, Tsukita S, Ebizuka Y, Sankawa U. 1987. Purification and properties of dihydrogeodin oxidase from Aspergillus terreus. J. Biochem. 101: 11-18.

28. Sasaki K, Mito K, Ohara K, Yamamoto H, Yazaki K. 2008. Cloning and characterization of naringenin 8-prenyltransferase, a flavonoid-specific prenyltransferase of Sophora flavescens. Plant Physiol. 146: 1075-1084.

29. Akashi T, Sasaki K, Aoki T, Ayabe S, Yazaki K. 2009. Molecular cloning and characterization of a cDNA for pterocarpan 4-dimethylallyltransferase catalyzing the key prenylation step in the biosynthesis of glyceollin, a soybean phytoalexin. Plant Physiol. 149: 683-693. 